\title{
Q MULTIMEDiales LERNEN ALS ANFORDERUNG ZUKUNFTSORIENTIERTER ERWACHSENENBILDUNG
}

\author{
Wolfgang Jütte
}

\section{KLÄRUNG DES SELBSTVERSTÄNDNISSES}

Die Schnelligkeit technologischer Entwicklung führt im allgemeinen zu einem "Hinterherhinken" der kulturellen Entwicklung. Derzeit klafft zwischen dem allseits aufgestellten Anforderungskatalog im Bereich Multimedia und den tatsächlichen Kompetenzen eine breite Lücke. Erst nachfolgende Generationen werden mit Medienkompetenz als Teil der Schulausbildung aufwachsen. Erwachsene Lernende müssen dagegen in eigener Regie kompensatorisch auf diese Handlungserfordernisse reagieren und entsprechende Fähigkeiten erwerben. Für Weiterbildungsinstitutionen eröffnet sich hier ein neues Bedarfsfeld.

Der durch neue Medien hervorgerufenen Wandel kann als paradigmatisch bezeichnet werden. Dies zeigt das Beispiel Internet, das sich zu einem globalen, exponentiell wachsenden Kommunikationssystem entwickelt. Wie kaum ein anderer Bereich reflektiert das Netz der Netze die heranziehende Wissens- und Informationsgesellschaft. Das Internet als Massenmedium wird vermutlich grundlegend die Formen der Kommunikation verändern, so wie es bisher der Buchdruck und das Telefon getan haben. Auch wenn man die Verheißungen neuer Medien mit Skepsis begegnet, so könnten sie weitreichende Konsequenzen für den Bereich des Lernens und Lehrens 
haben. Dies verlangt von der Erwachsenenbildung/Weiterbildung eine Selbstvergewisserung und Klärung des eigenen Selbstverständnisses.

Neue Medien verlangen ein erweitertes Anforderungsprofil von den zentralen Akteuren im Lerngeschehen:

- den Lernenden

- den Lehrenden (und)

- den Einrichtungen.

Wie werden institutionalisierte Bildungsträger mit den neuen Lernmedien umgehen? Dabei wird insbesondere der "Beziehungsaspekt" der neuen Medien aufgegriffen. Wie wird sich das pädagogische Rollenmuster zwischen Lehrenden und Lernenden wandeln?

Bei Trendanalysen und Zukunftsszenarien stellt sich das grundsätzliche Problem, dass das "Potenzial" zukünftiger Entwicklung betont wird. Dabei werden seitenlange Anforderungskataloge erstellt, ohne dass die Aktualität und Realisierungschancen genügend und kritisch betrachtet werden. So verheißend die Nutzungspotenziale sind, so unbefriedigend ist die gegenwärtige Situation. Trotz gelungener Modellprojekte überwiegen konzeptionelle pädagogische Defizite.

In der Aus- und Weiterbildung werden neue Medien in den nächsten Jahren eine zunehmend wichtige Rolle spielen. Hierbei eröffnen multimediale Lernformen neue Chancen; zugleich stellen sie eine Herausforderung für die pädagogische Arbeit da. Werden multimediale Lernumgebungen in Verbindung mit der Konzeption des selbstgesteuerten lebenslangen Lernens eine zentrale Rollen übernehmen? Wie werden sich zukunftsorientierte Strukturen der Erwachsenenbildung in der Wissensgesellschaft entwickeln? Wird sich eine neue Kultur des Lehrens und Lernens herausbilden?

\section{WISSENSGESELLSCHAFT, LEBENSLANGES LERNEN UND SELBSTGESTEUERTES LERNEN ALS KONZEPTIONELLER RAHMEN}

"Wissensgesellschaft", "lebenslanges Lernen" und "selbstgesteuertes Lernen" sind aktuelle Schlagworte, wenn es um zukunftsorientierte Formen des Lehrens und Lernens geht. Diese Konzepte, die eineinandergreifen, bilden zumeist den Referenzrahmen für den Einsatz neuer Kommunikationstechnologien. Es gibt eine Beziehungsstruktur zwischen multimedialen Lernprozessen und den 
Anforderungen an die Bildung in der Wissensgesellschaft, der Notwendigkeit lebenslangen Lernen und der Fähigkeit zum selbstgesteuerten Lernen. Lebenslanges und selbstgesteuertes Lernen wird als eine Antwort auf die sich herausbildende Wissensgesellschaft gesehen.

\section{WISSENSGESELLSCHAFT}

Gesellschaften befinden sich ständig im Wandel und werden mit bestimmten Attributen belegt. Während im Kontext der Verbreitung der Informations- und Kommunikationstechnologien lange der Begriff der "Informationsgesellschaft" vorherrschend war, rückt zunehmend das Leitbild einer "Wissensgesellschaft" in den Vordergrund. Sie ist dadurch gekennzeichnet, dass Wissen an Bedeutung gewinnt und zu einem zentralen Produktionsfaktor wie Arbeit, Boden und Kapital wird. In der Wissensgesellschaft wird der Umgang mit den Informations-und Kommunikationsmedien, nicht nur im Arbeits- und Berufsleben eine Schlüsselkompetenz sein. Die Wissensgesellschaft ist auf die Mündigkeit selbstverantwortlich lernender Individuen angewiesen. Ihnen ist es zur Aufgabe aus der unbegrenzt erscheinenden Informationsflut relevantes Wissen zu konstruieren.

In der Delphi-Befragung "Potenziale und Dimensionen der Wissensgesellschaft -Auswirkung auf Bildungsprozesse und Bildungsstrukturen" (1998), wird prognostiziert, dass die Entwicklung hin zur Wissensgesellschaft auch zu umgreifenden Veränderungen im Bildungssystem führt. Dies wird auch für die Aufgaben und Rollen der am Bildungsprozess beteiligten Akteure, den Lehrenden und Lernenden, nicht folgenlos bleiben. Insbesondere verschieben sich die herkömmlichen Rollenverteilungen zwischen Lehrenden und Lemenden.

\section{LEBENSLANGES LERNEN ALS BILDUNGSPOLITISCHE ANTWORT}

Noch nie in der menschlichen Geschichte sind Wissen und Fachkenntnisse so rasch veraltet wie heute. Das in der Schule erworbene Wissen reicht nicht mehr aus, um mit den gesellschaftlichen Veränderungen Schritt zu halten. Die Aneignung von Wissen wird zu einem ständig fortdauernden Prozess, der nicht mit dem Abschluss einer schulischen oder beruflichen Ausbildung beendet wird. Lernen wird zunehmend zu einem lebenslangen Bemühen. Mit dem lebenslangen Lernen wird die Vorstellung abgelöst, dass Lernen mit dem Ausbildungsende aufhört.

Das Konzept des lebenslangen Lernens hat auf nationaler und internationaler Ebene Konjunktur und wird als eine bildungspolitische Herausforderung des 21. Jahrhunderts gesehen. Wie ein roter Faden zieht 
sich durch jüngere bildungspolitische Stellungnahmen, Denkschriften und Entschließungen internationaler Organisationen der Gedanke des lebenslangen Lernens. Bildungspolitik wird zunehmend auf das strategische Konzept des "lebenslangen Lernens" ausgerichtet; das Bildungswesen soll zu einem "System lebenslangen Lernens" weiter entwickelt werden.

\section{SELBSTGESTEUERTES LERNEN}

Das Konzept des "Selbstgesteuerten Lernens" ist vielschtig (vgl. Weber 1996). Der Begriff "Selbstgesteuertes Lernen" wurde wie "lebenslanges Lernen" schon in den 70er Jahren entwickelt, aber durch die nachhaltige Nutzung multimedialer Kommunikationsmedien kommt ihnen eine neue Bedeutung zu. So wird Selbstlernen häufig in seiner multimedialen Variante diskutiert (s. Schöll/Passens 1998 und Stadelhofer 1999). Die Attraktivität der Konzeptes liegt aus erwachsenenpädagogischer Sicht in der Betonung auf Mündigkeit. Selbstgesteuertes Lernen lässt dem erwachsenen Lerner die Option für die adäquate Wahl seiner Lernpräferenzen offen und legt die Verantwortung für den Lernerfolg in die Hände des Lerners als agierendem Mitgestalter seiner Weiterbildung.

Zugleich ist man geneigt, der Versuchung zu erliegen, das Konzept des selbstgesteuerten Lernens als eine "Allround-Lösung" für pädagogische Probleme zu betrachten und gleichsam naiv über reale Grenzen und Schwierigkeiten hinwegzusehen.

Da ist zunächst die Gefahr der Verantwortungsverschiebung hin zu den Lernenden. Das Konzept der Selbstverantwortung im Lernprozess kann dazu genutzt werden, finanziellen Einsparungsabsichten als Alibifunktion zu dienen. Aus der Förderung selbstgesteuerten und lebenslangen Lernens darf aber kein Rückzug aus der öffentlichen Mitverantwortung für Weiterbildung abgeleitet werden (vgl. Krug 1997, S. 86). In Verbindung mit multimedialen Lernumgebungen bedeutet dies, dass auch für Personen mit geringer Weiterbildungsbereitschaft und sozial Benachteiligte bildungspolitische Lösungen gefunden werden müssen. Sonst droht die Gefahr der Segmentierung mit den Folgen, dass Kompetenzerweiterungen zu einer elitären Bildungsoption für finanziell besser gestellte wird.

\section{MULTIMEDIALES LERNEN-MERKMALE UND FORMEN}

"Multimedia" bezeichnet zumeist das Zusammenwachsen verschiedener Medien (Telefon, Computer und Fernsehen) und die daraus neu entstandenen 
Nutzungsmöglichkeiten. Der unscharfe Begriff "Multimedia" ist mittlerweile weit mehr als ein technischer Begriff, als Sammelbegriff avanciert er zu einer neuen "Zauberformel".

Wenn die spezifischen Leistungen von Multimedia für Lehr- und Lernprozesse betont werden, dann besteht zumeist die Gefahr, dass Multimedia mit naiven Lernannahmen in Verbindung gebracht werden (Weidenmann 1997, S. 80). Diese beruhen meistens auf einer Summationstheorie, d.h. es wird angenommen je mehr Sinnesmodalitäten angesprochen werden, um so höher sei der Lerneffekt. Als die drei häufig verbreitetsten naiven Annahmen sieht er die folgenden Aussagen :

1. Multimedia spricht mehrere Sinneskanäle an; das verbessert das Behalten.

2. Die Abwechslung in der Darbietung des Lernmaterials motiviert die Lerner.

3. Multimedia aktiviert die Lerner.

Diese Aussagen alleine können den tatsächlichen Nutzwert von Multimedia-Anwendungen nicht erfassen. Vielmehr liegt ihr Vorteil in der spezifischen Auswahl, die bei der didaktischen Konstruktion zur Verfügung stehen. Bei der Erstellung der entsprechenden Lernumgebung können dem Lernenden verschiedene Angebote der Wissensdarbietung optional zur Verfügung gestellt werden. Dabei spielt allerdings die didaktische Umsetzung die größte Rolle für den Lernerfolg, denn ohne einen gezielten und strukturell durchdachten Einsatz können auch in Multimedia-Anwendungen mit Texten, Diagrammen und Abbildern keine positiven Lernresultate erzielt werden.

Diese werden vielmehr entscheidend durch die Fertigkeiten und Fähigkeiten bestimmt, die die Lernenden mitbringen. Ohne die interpretierenden Handlungen des Lernenden ist eine Hypermedia- Basis nur ein großes Warenhaus von Texten, Tönen, Bildern, Filmen und Animationen. Deshalb beinhalten Lernprogramme oftmals eine tutorielle Komponente, die dem Benutzer Orientierungshilfen an die Hand geben und Navigationsprobleme verringern bzw. ausschließen sollen (vgl. Haack 1997, S. 155).

Das Internet lässt sich nicht direkt als Lernmedium bezeichnen, da es die Lernenden nicht mit vorgegebenen Lernzielen, Arbeitsschritten und Leistungskontrollen konfrontiert. Vielmehr kann das Netz als kognitives Werkzeug betrachtet werden, das die Auseinandersetzung mit verschiedensten Inhalten anregt, motiviert, unterstützt, erleichtert und korrigiert und somit individuelles und selbstgesteuertes Lernen begünstigt (vgl. Döring 1997, S. 321). 


\section{MULTIMEDIALE LERNUMGEBUNGEN FÜR SELBSTGESTEUERTES LERNEN}

Multimedia kann Lernen genau in diese Spur der Individualität zurückbringen. Die Bedürfnisse des einzelnen können besser berücksichtigt werden als dies in den herkömmlichen Lehr- Lernsituationen möglich war. Multimedia eröffnet die Möglichkeit, einen spezifischen und individuellen Lernweg anbieten zu können; sei es über multimediale Softwareprogramme, über das Internet oder auch in virtuellen Welten. Es hängt mit von den Beschaffenheiten multimedialer Lernumgebungen $a b$, inwieweit sie selbstgesteuertes Lernen anregen, unterstützen und fördern. Unter Lernumgebung kann die Gesamtheit des Arrangements verstanden werden, das die Lernenden umgibt. Nach Friedrich/Mandl (1997, S. 258) sind dabei die äußeren Lernbedingungen (Personen und Institutionen, Geräte und Objekte, Symbole und Medien, Informationsmittel und Werkzeuge) und die Instruktionsmaßnahmen (Lernaufgaben, Sequenz der Lernschritte, Methoden, u.a.) von entscheidender Bedeutung, um Lernen zu ermöglichen und zu erleichtern.

Computerunterstützte Lernumgebungen eignen sich nicht nur als vorfabrizierte, von den Lernenden nicht mehr modifizierbare "Instruktionsmedien"; sie eignen sich auch als eigenaktivitätssteigernde "Konstruktionsmedien". Entsprechend lassen sich computerbasierte Lernumgebungen in expositorische und explorative Designs unterscheiden, in denen die Aspekte der Selbststeuerung von unterschiedlichen Lernzielintentionen ausgehen (vgl. Niegemann 1998).

Expositorische (darbietende) Unterrichtskonzepte zeichnen sich durch operationale Lernziele, kleine Informationseinheiten, eine aktive Rolle der Lernenden und eine unmittelbare Rückmeldung aus. Elemente der Selbststeuerung beschränken sich auf die Unabhängigkeit von einer lehrenden Person, auf die Regulation von Lernzeiten und auf die internen Verarbeitungsprozesse bei der Rezeption der vorbereiteten Lerninhalte. Beispiele sind "drill and practice"-Programme mit Übungsfunktionen und interaktive tutorielle Lernsysteme zur Vermittlung neuer Lehrstoffe (vgl. Friedrich/Mandl 1997, S. 258).

Den explorativen multimedialen Lernumgebungen ist dagegen gemeinsam, dass sie von den Lernenden tendenziell mehr und auch komplexere Eigenaktivitäten verlangen. Dazu zählen

- Multimedia- Lernumgebungen, die die Möglichkeit bieten, das Lerntempo und die Lernzeit selbst zu steuern und den Zugriff auf diverse Darstellungsformen anbieten, wie z.B. geschriebene und gesprochene Sprache, stehende und bewegte Grafiken, Bilder oder Videosequenzen, 
- Simulations- und Modellbildungssysteme zum Training komplexer Handlungsabläufe,

- Hypermedien und Hypertext, die aufgrund der nichtlinearen Verknüpfungsmöglichkeiten den Lernenden große Freiräume bei der Auswahl der Lernschritte eröffnen und den Aufbau multipler Perspektiven unterstützen (vgl. Friedrich/Mandl 1997, S. 266).

Das Maximum der Selbststeuerungsmöglichkeiten kann aber nicht per se die pädagogische Zielsetzung sein. Denn bei fehlender Unterstützung und komplexen Selbststeuerungsdesigns drohen kognitive Überlastungen der Lernenden. Vielmehr sollte der Grad der Selbststeuerungsmöglichkeit von den jeweiligen ausgeprägten Lernstrategien abhängen.

\section{NEUE ANFORDERUNGEN UND ÜBERFORDERUNGEN}

Im Kontext neuer Medien wird zumeist das "Spielerische" betont. Nicht umsonst gilt "Convenience" (Bequemlichkeit) als Schlüsselbegriff des Internets. Anstelle der "Anstrengung des Begriffs" lade das Netz zum Flanieren ein. Das neue Medium suggeriere, es käme nur darauf an, Wissen zu finden und abzurufen. Die Produktion von Wissen vollziehe sich durch die scheinbare Arbeit der Finger auf der Tastatur.

Neue Medien verlangen jedoch sich auf neue Formen des Lernen einzulassen. Dies führt zu zahlreichen "Anforderungen", die einzelne auch als Zumutungen empfinden. Erwachsenenbildung muss hier sensibel für Überforderungen sein.

Multimediales Lernen in selbstgesteuerter Form verspricht einerseits ein hohes Gewinn-potenzial für die Aus- und Weiterbildung, verlangt andererseits aber ein verändertes Anforderungsprofil, sowohl von den Lernenden als auch den pädagogischen Fachkräften und den Institutionen, damit selbstgesteuertes Lernen bewältigt werden kann.

\subsection{ANFORDERUNGEN AN TEILNEHMER}

In den Szenarien der Wissensgesellschaft wird von einem selbstständigen Lernenden ausgegangen, der hochgradig aktiv bei der Aneignung von Wissen ist; der sowohl die relevanten Fragen selbst stellt als auch in eigener Regie Antworten darauf findet. So heißt es im Delphi-Report:

- "Die Rolle des Lernenden (...) wandelt sich immer mehr hin zu der eines eigeninitiativen ,Entdeckungsreisenden', der im Team mit 
anderen, mit Forschergeist, Neugier und der Unterstützung Lehrender neues Wissensterrain erobert und erschließt. Lernende agieren im Rahmen vereinbarter Aufgabenfelder als selbstverantwortliche ,Forscher und steuern in diesem Rahmen inhaltliche Schwerpunkte, methodische Angänge und das Tempo ihres Lernens zum Teil selbst.

- Lernende fungieren immer weniger als passive Rezipienten bzw. ,Wiederkäuer" vorgegebener Lerninhalte und immer mehr als aktive Gestalter von Lernprozessen und Lerngruppen, in denen sie projektbezogen oder phasenweise auch die Rolle des Lehrenden übernehmen." (Delphi-Befragung 1998, S. 73)

Lernen wird oftmals mit Lehrer- bzw. Dozentenorientierten Veranstaltungen verbunden, die in Form von Frontalunterricht in Lehrermonologen abgehalten werden. Teilnehmern fällt es häufig schwer, Lernen als einen aktiven Prozess zu begreifen, der über die pure Wissensvermittlung hinausgeht. Obwohl Teilnehmer in Weiterbildungsveranstaltungen erwarten, dass sie ihrem Status als Erwachsene entsprechend behandelt werden, kann gleichzeitig die Erwartung nach einem direktiven Führungsstil bestehen. Offene Seminarstrukturen, die die Lernenden von der passiv-rezipierenden Rolle zu mehr Eigenaktivität hinführen sollen, stoßen auf Skepsis oder sogar Ablehnung.

Jeder Erwachsene hat sich im Laufe seiner Biografie in vielfältiger Form mit Lernen auseinandergesetzt und daraufhin ein individuelles Lernkonzept entwickelt. Die bereits vorhandenen Strategien, beruhend meistens auf Schulerfahrungen, haben sich in irgendeiner Form als "brauchbar" erwiesen. Von den Teilnehmern wird nun ein verändertes Lernverhalten verlangt, das sich oftmals an die bereits verinnerlichten Lernstrategien nicht nahtlos anknüpfen lässt. Die Bereitschaft, sich mit multimedialem Lernen auseinanderzusetzen und sich auf neue Lernerfahrungen einzulassen, erfordert ein hohes $\mathrm{Maß}$ an Offenheit. Subjektive Ängste können den konstruktiven Umgang mit dem Computer versperren.

Die Hinführung zu mehr Selbststeuerung im Lernprozess und die Loslösung vom dozentenorientierten Lernen ist für jeden Lernenden eine unterschiedlich hohe "Hürde", die nicht kurzfristig zu überwinden ist. Letztlich kann dieser Wechsel aber zu einem selbstverständlicheren Umgang mit Bildung führen und die Verantwortung über den Lernerfolg zu den Lernenden hinlenken.

\section{Medienkompetenz und "Selbstlern-Management-Kompetenz"}

Die Teilnehmer in der Erwachsenenbildung sind zwar in erheblichen Maß an den konsumtiven Umgang mit Medien gewöhnt, sie besitzen aber bei weitem nicht in gleicher Weise Erfahrungen mit Medien als Lehr-und 
Lernmittel. Wenn ihnen Medien in ihrem bisherigen Bildungsverlauf begegnet sind, dann in der Form, dass mit ihnen Informationen "verabreicht" wurden, die den Lernenden in ausschließlich passivrezipierender Rolle beließen (vgl. Hüther 1994, S. 295).

Im Kontext der Neuen Medien wird der Begriff der Medienkompetenz als Schlüsselbegriff neu diskutiert (vgl. von Rein 1996). Medienkompetenz bedeutet im engeren Sinne die Fähigkeit, mit Komplexität umgehen zu können, wie sie beispielsweise im Hypertextsystemen angeboten werden. Dies erfordert vom Lernenden eine adäquate Rezeptionsform. Daten müssen aus großen Datenmengen ausgewählt, analysiert und interpretiert werden; dafür ist sowohl strategisches Wissen als auch ein gewisses Maß an Entscheidungsfähigkeit erforderlich. Medienbotschaften aus dem Internet machen eine erweiterte und veränderte Kommunikationskompetenz erforderlich (vgl. Gabriel 1997, S. 167).

Der kompetente Umgang mit den Neuen Medien setzt auf die Selbstorganisationspotenziale. Es handelt sich um umfassende Selbstlernkompetenzen. Dies verlangt von Teilnehmern eine "SelbstlernManagement-Kompetenz" zu entwickeln, d.h.:.

"ein Bewußtsein zu haben und zu aktivieren

- was zu lernen wichtig ist und was nicht (Relativierungsfähigkeit),

- was man bereits kann und weiß (Individuelle Kompetenzen/ Qualifikationen),

- wohin man sich entwickeln will (eigene Lerninteressen/Lernziele),

- wo man sich gesellschaftliche Orientierungen holen kann, was zu lernen wichtig wäre (Lernbedarfe),

- wie man subjektive Lernbedarfsanalysen macht,

- wie man selbst am besten/effektivsten lernt (Lernstrategien/Bewußtsein über die inneren und äußeren Bedingungen, die das eigene Lernen fördern/behindern),

- wo die eigenen Grenzen liegen." (Corcilius/Dedorath/Müller-Sämann 1998, S. 99)

\subsection{ANFORDERUNGEN AN LEHRENDE}

Für die Lehrenden der Erwachsenen- und Weiterbildung bedeuten der Einzug multimedial gestützter Lehr- und Lernsysteme und veränderten 
Lernanlässen eine Auseinandersetzung mit ihrem eigenen DozentenSelbstverständnis und der Übernahmen eines Rollenwechsels:

- "Die Rolle der Lehrenden tendiert hin zu der von Beratern, die im Prozess der Wissensaneignung Informationen geben, darüber hinaus aber vor allem anregen, unterstützen und zu Selbstverantwortung und Eigenständigkeit animieren. Ihre Rolle als Vermittler von Fachwissen tritt dabei stärker in den Hintergrund.

- Lehrende werden zu Moderatoren von Lernprozessen in Gruppen; sie sind weniger Fachautorität als vielmehr diejenigen, die den Wissensund Kompetenzenerwerb in Lernteams organisieren und begleiten, die den Austausch zwischen Lernenden untereinander, aber auch mit den Lehrenden und schließlich den Austausch zwischen den Lehrenden selbst fördern." (Delphi-Befragung 1998, S. 74)

Wenn sich Aus- und Weiterbildner weniger als Wissensvermittler und mehr als kommunikationsstarke Experten mit den Aufgaben der Lernmotivation und -beratung sehen, dann ist dies auch für hierarchische Verhältnis zwischen Lehrenden und Lernenden nicht folgenlos. Als Moderator, Facilitator oder Vermittler steht ihnen keine übergeordnete Position mehr zu, sondern die des beratenden Begleiters auf derselben Ebene. Vielmehr müssen sie anerkennen, dass sie nicht mehr auf allen Fachgebieten Fachleute sein können; sondern dass sie zumeist in neuen Bereichen auch Lernende seien müssen. Zugespitzt formuliert hieße dies: Wer Nicht-Wissen zu erkennen gibt, leistet einen Beitrag zur Lernkultur. Wenn Erbhöfe und Monopolstellungen des Wissens verloren gehen, dann ist dies mit hierarchischen Organisationsstrukturen nur schlecht zu vereinbaren.

\section{Veränderung des Professionsverständnis und neue pädagogische Berufsbilder}

Mit der schnellen Entwicklung der Telekommunikationstechnologien wird auch ein Wandel von Berufsbildern prognostiziert. Neue Beschäftigungsfelder entstehen an den Schnittstellen bisher getrennter Bereiche/Berufe. Neue Wortschöpfungen und Etiketten zeigen nicht nur neue Berufsbilder von Lehrenden auf, sondern verweisen zugleich auf den tiefergehende Wandel in den pädagogischen Leitbildern. Im folgenden haben wir eine Liste von vorgefundenen Begriffen der Diskussion aufgelistet. [Die meisten erklären sich von selbst; einige wurden stichwortartig erläutert.]:

- Animator

- Arrangeur von Lernumgebungen 
- Autor von Selbstlernmaterialien

- Berater, Lernberater

- Beobachter

- Bildungsmanager

- Coach; Net-Coach, Inhouse-Coach.

- Change agent

- Content-Adapter (Anpasser von Lern-Inhalten)

- Facilitator

- Fallberater

- Initiator von Lernprozessen

- Info-Broker, (Beschaffer von Informationen)

- Kommunikations-Designer

- (Lebensbezogener) Lernexperte

- Lernpartnerschafts-Helfer

- Lernspielentwickler

- Lernsteuerungstrainer

- Lernvermittler

- Mentor

- Moderator, Lernmoderator, Online-Moderator

- Motivator

- Multimedia-Trainer

- Navigator

- Organisator

- Prozessbegleiter

- Supporter von Lernprozessen

- Tele-Tutor, Tele-Lernbegleiter

- Transfer-Begleiter

Diese Begriffe spiegeln die Veränderungen des Berufsbildes wieder. Anstelle des Dozenten und Trainers als Sender von Wissen tritt der Lernbegleiter, der Lernprozesse anregt und beratend dem Lernenden als Entdeckungsreisenden zur Seite steht. Von pädagogischen Mitarbeitern und Wissensexperten wird weniger die reine Wissensvermittlung erwartet, sondern eher die Fähigkeit, auf Ressourcen hinzuweisen, Strategien 
aufzuzeigen, für methodische Vorgänge zu sensibilisieren und das Gefühl für konstruktive Veränderungen zu wecken.

Die Lehr-/Lernsituationen sollten nicht mehr dozentenorientiert gestaltet werden, sondern eher die Momente fokussieren, in denen die Teilnehmer sich aktiv und konstruktiv mit dem Lerninhalt auseinandersetzen müssen. Auf diese Weise können sie das nötige Selbstbewusstsein für immer mehr Selbststeuerung im Lernprozess erwerben. Die Lehrenden brauchen das Gespür für notwendige und angebrachte Hilfestellungen, um einerseits dem Wunsch nach Begleitung nachzukommen und um andererseits sich zum angemessenen Zeitpunkt aus dem Lernprozess zurückzuziehen. Nach der schrittweisen Hinführung zu mehr Selbststeuerung sollte die pädagogische Betreuung dann optional zur Verfügung stehen.

\section{Flexible Rollenverteilung zwischen Lehrenden und Lernenden}

Die Rolle des Lehrenden wird nicht obsolet, aber sie wird flexibler gehandhabt werden:

"In einer flexibleren Rollenverteilung zwischen Lehrenden und Lernenden gewinnen zudem Patenschaftsmodelle bzw. Mentoringkonzepte an Bedeutung. Lehrende, (Vor-) Schüler, Auszubildende, Studenten oder Teilnehmer an Fortund Weiterbildung lernen jeweils miteinander und voneinander. Eine Aufgabe der Lehrenden liegt in der Anregung von Patenschaften bzw. Mentoring innerhalb der Gruppe von Lernenden, aber auch durch Mentoren von außerhalb, indem zum Beispiel ältere Schüler als Ansprechpartner von jüngeren fungieren, Mentoren aus der Wirtschaft Auszubildende oder Studenten betreuen, usw." (Delphi-Befragung 1998, S. 74)

Damit wird auch das Lernen im Team als wechselseitige Unterstützung und die Bildung kommunikativer Lernpartnerschaften bedeutsam. Hinzu kommen neue Kooperationspartner mit ins Boot. Unterschiedliche gesellschaftliche Gruppen, wie Bürgerinitiativen oder Intereressengemeinschaften, werden stärker miteinbezogen.

\section{Konsequenzen für Aus- und Fortbildung}

Selbstgesteuertes Lernen initiiert eine Akzentverschiebung der professionellen Handlungskompetenzen. Auf diese veränderten Rollen müssen Kurs- und Seminarleiter vorbereitet werden. Ein Schlüssel liegt neben der Ausbildung in einer berufsbegleitenden Fortbildung der Aus- und Weiterbildner.

Widerstände, Skepsis und technische Berührungsängste gegenüber multimedialen Lernprogrammen bestehen nicht nur auf Seiten der 
Teilnehmerschaft, sondern auch beim Lehrpersonal. Attraktiv gestaltete Computer- Lernprogramme implementieren einen hohen Qualitätsstandard der Wissensvermittlung. Hier sehen sich einige Dozenten in einer Konkurrenzsituation. Hinzu kommt, dass Kurs- und Seminarleiter wenig Erfahrungen auf dem Gebiet der Computertechnologie haben und sich unsicher fühlen. Durch Fortbildungen können sie Sicherheit im Umgang mit Computerlernprogrammen im Internet erlangen und einen Blick für die didaktischen und gestalterischen Möglichkeiten der neuen Medien gewinnen.

Der Umfang der Herausforderung, die sich mit multimedialem Lernen stellt, ist jedoch viel umfassender, als sich lediglich auf den Einsatz neuer multimedial gestützte Lehr- und Lernsysteme vorzubereiten. Es kommt nicht zu einer Funktionsminderung der Lehrenden, ihr pädagogisches know how ist auch weiterhin gefragt (vgl. Tietgens 1998, S. 42f.). Vielmehr werden neue Kompetenzanforderungen hinzukommen, um mit der Komplexität umzugehen:

"Dazu gehört auf der Seite der Lehrenden, dass sie zunehmend sowohl lehrend als auch beratend gefordert sind. Das hinwiederum erfordert im face to face Umgang andere Kompetenzen als in der Online-Bildung. Das von den individuellen Lernern hinwiederum erwartete Arrangement offener und damit individualisierender Lernmöglichkeiten übersteigt sehr oft die Kompetenzen des einzelnen Lehrenden usw." (Weinberg 1998, S. 10).

Fortbildungsangebote zur Erlangung von Medien-Kompetenz müssen verschiedene Kompetenzebenen differenzieren. Stang/Hagedorn/Nispel (1998, S. 123f.) unterscheiden die folgenden:

"Wahrnehmungskompetenz: Erfahrung mit der Entschlüsselung von Medien in ihren vielfältigen Varianten (Konstruktivität);

Reflexionskompetenz: Hintergrundwissen über Strukturen, Funktionsweisen, Entwicklungen des Mediensystems, seine Programme sowie seine vielfältigen ökonomischen, sozialen, kulturellen und politischen Bezüge;

Diskurs-Kompetenz: Fähigkeit zur reflektierten Teilnahme am öffentlichen Diskurs über Medien und - mit Hilfe neuer Medienkommunikation (Newsgroups etc.) - zur Teilnahme am Mediendiskurs;

Medientechnische Kompetenz: Offline- und Online-Anwendungen unterschiedlicher Intensität und Reichweite kennen und bezüglich der Relevanz für die eigenen Arbeitsfelder einschätzen können; Anwendungskompetenzen in einem zu definierenden Ausschnitt erwerben, z.B. Hypertext-Gestaltung;

Methodisch-didaktische Kompetenz: Gestaltung von Lernarrangements, die neue Medien integrieren; 
Medien-kommunikative Kompetenz: E-Mail-Kommunikation; Tele-Tutoring, Tele-Moderation etc.;

Pädagogisch-soziale Kompetenz: Neues Selbstverständnis, neue Rollen und Funktionen (Moderatorin, Coach, Beraterin etc.);

Organisationskompetenz: Integration der Mediennutzung in organisatorische und institutionelle Entwicklungsprozesse (Medien-Management):

Fachkompetenz: Überblick über die für die eigenen fachlichen Inhalte relevanten Möglichkeiten multimedialer Unterstützung (Kenntnisse von Lernsoftware, WWW-Unterstützung in inhaltlicher und methodischdidaktischer Dimension)."

\subsection{WANDEL DER INSTITUTIONEN}

Seitdem sich in der Weiterbildung die Akzentsetzung stärker hin zum selbstgesteuerten Lernprozess verschiebt, stellt sich die Frage nach den Aufgaben institutioneller Bildung. Weiterbildungsinstitutionen müssen sich mit einer Neu- bzw. Erweiterungsdefinition ihrer Aufgaben und Ziele befassen.

Auch wenn das selbstgesteuerte Lernen auf den ersten Blick eine stärkere Eigenverantwortlichkeit der Lernenden impliziert, bedeutet dies nicht, dass Bildungsinstitutionen überflüssig werden. Aber organisierte Weiterbildungsträger müssen sich mit einer institutionellen Entgrenzung auseinandersetzen. Bildungs- und Lernprozesse Erwachsener finden immer mehr außerhalb pädagogischer Institutionen und jenseits professioneller Betreuung statt. Das bedeutet nun nicht, dass Bildung sich zukünftig nur in pädagogisch noch nicht erfassten Handlungskontexten und Lebensbereichen abspielt, vielmehr entstehen neue Mischungsverhältnisse zwischen pädagogisch und nicht-pädagogisch strukturierter Aneignung (Kade 1997, S. 21).

Das institutionelle Selbstbild wird zukünftig nicht mehr lediglich auf den klassischen Lehr-Lernsituationen beruhen; vielmehr wird die Konzeptstruktur offener gestaltet und somit Raum für selbstgesteuerte Lernprozesse und multimediale Arrangements geschaffen werden. Mediale Formen der Wissensaneignung gewinnen immer mehr an Bedeutung .

Die professionelle pädagogische Begleitung nimmt eine wichtige Rolle ein, damit eine selbstgesteuerte Lernkultur sich entwickeln kann. Das Ziel der Lernkompetenzförderung, d.h. weg von dem Konsumentenstatus hin zu aktiver Teilnahme, ist nur mit Hilfe institutioneller Weiterbildung zu erreichen. Hier eröffnen sich neue institutionelle Entwicklungsaufgaben, die aber nicht kurzfristig umsetzbar sind. 
Zusammenfassend können die veränderten Aufgabenkomplexe der institutionellen Weiterbildung lauten:

- Institutionalisierte Lern- und Bildungsangebote müssen sich mit Konkurrenzsituationen auf einem offenem "Teilnehmermarkt" auseinandersetzen und das Prinzip der Selbstorganisation als Schlüsselkonzept in die Institutionsstruktur mit einflechten.

- Bildungsinstitutionen müssen komplementäre Wechsel- und Ergänzungsbeziehungen zu informellen Lernformen entwickeln. Es sind systematische Strukturen notwendig, die dem zufälliganlassbezogenen informellen Lernen gerecht werden und eine sensible und professionelle Unterstützung, Weiterführung und Ergänzung für den Lernenden darstellen.

- Mit Hilfe Neuer Medien könnten flexiblere Seminarstrukturen angeboten werden, die den individuellen Anforderungen der Lernenden entsprechen.

- Selbstgesteuertes Lernen erfordert - gerade für Lernende mit schwach ausgeprägten Lernstrategien - eine optional zur Verfügung gestellte pädagogische Betreuung und darf nicht als "Selbstgänger" eingestuft werden.

- Anstelle einer fachlich eingegrenzten Wissensvermittlung ist eine Hinführung zur aktiven und breitgefächerten Auseinandersetzung mit Bildung und Lernen gefragt ("Lernen zu Lernen").

\section{FÜR EINE NEUE KULTUR DES LERNENS}

Neue Medien bedeuten nicht zwangsläufig neues Lernen. Auch sie können einer traditionellen Lernphilosophie anhängen, die es dann nur erlaubt "vom neuen Wein in alten Schläuchen" zu sprechen.

Selbstgesteuerte Lernkonzepte als tragfähige Option im Bildungswesen sind keine "Selbstgänger"; vielmehr mit pädagogischem Arbeitseinsatz verbunden. Die besondere Herausforderung besteht darin, eine vorwiegend passive, mit langer Tradition behaftete Lernkultur, auf eine aktive vorzubereiten.

In der traditionellen Aus- und Weiterbildung sind immer noch reine Wissensvermittlung, frontale Unterrichtsmethoden, starke Fächergrenzen, Wissensreproduktion auf ein bestimmtes Ziel hin, von außen angeleitete (extrinsische) Motivation etc. anzutreffen. Trotz Gruppenarbeit und Projektarbeit verläuft das Lehr-Lern-Geschehen seit Generationen in 
denselben starren Bahnen, und es dominiert eine passiv-rezipierende Form der Wissensaufnahme.

\section{SCHWÄCHEN TRADITIONELLER LERNPHILOSOPHIE}

Lehrende der Weiterbildung wurden je nach pädagogischem Selbstverständnis und den eingesetzten Feldern unterschiedlich bezeichnet: Ausbilder, Dozenten, Kursleiter, Moderatoren Teamer oder Trainer. Gemeinsam war aber den Lehrenden, dass sie als "Transporteur von Wissen" begriffen wurden. Dieses sich dahinter verbergende "Modell vom lehrenden Sender und lernenden Empfänger" (Delphi-Befragung 1998, S. 73), die Aufteilung in einen wissenden und unwissenden, in einen aktiven und passiven-rezeptiven Part, lässt sich weiterhin in der Bildungsarbeit beobachten. Alltagstheoretische Annahmen zum Lernen sind eng mit der Vorstellung vom "Input und Output" verbunden. Hinter dieser Vorstellung des Wissenstransports vom Wissen zum Unwissenden und der anschließenden Überprüfung steckt ein "Lehr-LernKurzschlussmodell". Die traditionelle Auffassung des Lernens, die häufig als "Trichter- oder Speichermodell" daherkommt, ist dabei nicht auf bestimmte Bildungseinrichtungen beschränkt.

Die neuen Medien fordern eine Revision der Lernkultur. Vor allem konstruktivistische Ansätze, die im Zusammenhang mit den neuen Medien verstärkt diskutiert werden, hinterfragen traditionelle Lernphilosophien (vgl. Siebert 1999). Aus konstruktivistischer Sicht wird den Lernenden eine aktive und konstruktive Rolle beim Wissenserwerb zugeschrieben, und Lehre wird als Anregung und Unterstützung der Eigenaktivität verstanden. Die Förderung einer selbstgesteuerten Lernkultur bedeutet zunächst ein wandelndes Lernbewusstsein und -verständnis bei allen Beteiligten zu entwickeln.

\section{NEUE INSTITUTIONELLE VERANTWORTUNG}

Das nachhaltige Interesse am selbstgesteuerten Lernen ist nicht nur Gründen der Innovation geschuldet, sondern auch der Hoffnung nach Kostenersparnissen. Dies gilt sowohl für den Bereich der öffentlich geförderten als auch der betrieblichen Weiterbildung. Hier wird Verantwortung für die Weiterqualifikation des einzelnen Mitarbeiters auf diesen selbst verschoben; der Arbeitgeber wird in seiner Pflicht entlastet, den Aufwand und die Kosten für die Weiterbildung zu übernehmen.

Die Betonung des selbstgesteuerten Lernkonzepts bedeutet nicht, die Lernenden mit ihrem Weiterbildungsschicksal alleine zu lassen. Wenngleich 
Erwachsene für sich selbst verantwortlich sind, wie offen sie mit Lernen und Bildung umgehen, liegt die Verantwortung für ein befriedigendes Lernresultat nicht allein auf die Schultern des Lernenden.

Weiterbildung als Gemeinschaftsaufgabe aller Beteiligten darf auch durch eine stärker betonte Selbststeuerung und Eigenverantwortung des Lerners ihren Anspruch an pädagogische Professionalität nicht verlieren. Pädagogische Arbeit darf sich nicht durch die These begründen, dass sie sich durch selbstgesteuerte Lernprozesse überflüssig macht. Motivationale, fachliche und materielle Voraussetzungen sind erforderlich, damit die Individuen auf selbstgesteuerte Lernprozesse bewusst zugreifen können.

Als konstitutive Rahmenbedingungen für selbstgesteuertes Lernen hat $\mathbf{P}$. Krug (1997, S. 88) folgende Punkte zusammengetragen:

- "Das subjektive Recht auf Weiterbildung, auf selbstgesteuertes Lernen muss durch objektive, öffentlich mitverantwortete Rahmenbedingungen abgesichert bleiben, insbesondere für benachteiligte Gruppen.

- Es bedarf eines organisierten Angebots von Selbstlernmöglichkeiten sowie von Einführungen zur Beherrschung dieser Selbstlernprozesse.

- Es bedarf darüber hinaus einer Ermöglichung eines offenen Zugangs $\mathrm{zu}$ solchen selbstorganisierten Selbstlernprozessen sowohl in materieller, technischer als auch in sozialer Hinsicht.

- Es bedarf schließlich einer hinreichenden Zeitsouveränität für selbstgesteuerte Lernprozesse am Arbeitsplatz, in der Freizeit und in der Bildungszeit als Mobilzeit.

- Es bedarf weiterhin des Qualitätsschutzes und der Werbung für Akzeptanz selbstgesteuerter Lernprozesse."

Institutionellen Weiterbildungsträgern steht auch weiterhin die Verantwortung zu, einen sinnstiftenden Rahmen für mehr Selbststeuerung im Lernprozess zu schaffen. Der institutionelle Rahmen bietet dem Lernenden die erste Anlaufstelle, um in betreuter Umgebung seine persönliche Medienkompetenz zu erarbeiten. Dem Lehrenden kommt dabei die Aufgabe zu, eine Lernatmosphäre zu schaffen, die den angstfreien selbstbewussten Umgang mit Bildung fördert und eine pädagogische Begleitung zu garantieren, die die Individualität der Teilnehmer berücksichtigt. Dazu gehört auch die Möglichkeit der individuellen Beratung.

Die Annahme, dass selbstgesteuertes Lernen und die stärkere Nutzung multimedialer Lernumgebungen langfristig der institutionellen Weiterbildung den Rang ablaufen, geht von konkurrierenden Positionen aus. Eine Deinstitutionalisierung des Weiterbildungssystems zählt nicht zu 
den erstrebenswerten Fernzielen. Vielmehr wird der institutionelle Rahmen auch weiterhin notwendig sein, um Weiterbildung für alle zugänglich zu machen.

Selbstgesteuertes Lernen und multimediale Lernumgebungen stehen nicht in einer unmittelbaren Konkurrenzsituation zur institutionellen Weiterbildung. Anstatt einer falschen Dichotomisierung soll auf die fruchtbaren Verbindungselemente zwischen diesen unterschiedlichen Bildungsparteien hingewiesen werden. Die Komponenten sollten wie Zahnräder ineinander gefügt sein und jeweils an den Punkten ansetzen, wo die Möglichkeiten der anderen ausgereizt sind. So kann sich ein System entwickeln, das auf die individuellen Bedürfnisse der Lernenden zugeschnitten ist. Wenn beispielsweise im institutionellen Rahmen Seminare über den Gebrauch von multimedialen Lernarrangements angeboten werden, kann durch die schrittweise Aneignung von Medienkompetenz das Interesse geweckt werden, selbstständig mit derartigen Programmen zu arbeiten.

\section{ENTSTEHEN VON LERN-NETZWERKEN DURCH BILDUNGSALLIANZEN}

Mit Flexibilität und Offenheit für eine Erweiterung der institutionellen Strukturen könnten die Institutionen den Ausgangspunkt für ein raumgreifendes und unfassendes System darstellen, das sich aus verschiedenen "Bildungs- Bausteinen" zusammensetzt.

Wenn es durch eine umfangreiche Zusammenarbeit aller Beteiligten möglich sein wird, ein Bildungsnetzwerk aufzubauen, das für jeden Lerner die adäquaten Lernstrategien anbieten kann, dann gehören multimediales und selbstgesteuertes Lernen zu wichtigen Bausteinen dieses Netzwerks. Dieses sollte kein konkurrierendes Bildungsnetzwerk, sondern eher ein kooperierendes System sein, das dazu auffordert, auf verschiedenen Wegen, die individuellen Lernkompetenzen selbstständig zu erweitern. Somit könnte durch Bildungsallianzen eine attraktive Lernlandschaft an verschiedenen Lernorten entstehen. Damit würden Lernarrangements geschaffen, die für jeden zugänglich sind und gleichzeitig mit pädagogischer und fachlicher Beratung, eine kompetente Betreuung anbieten.

\section{WEITERBILDUNGSEINRICHTUNGEN ALS BILDUNGSSTÜTZPUNKTE UND KOMMUNIKATIONSAGENTUREN}

Um den veränderten Lernanforderungen und -möglichkeiten Rechnung zu tragen, wird die bisherige Organisationsstruktur von Weiterbildungsinstitutionen 
neue Impulse aufnehmen müssen. An konzeptionellen Vorschlägen mangelt es nicht. So schlägt Dohmen (1999, S 71ff) den Aufbau eines Netzwerkes von kommunalen Lernservice-Stationen vor, den Weiterbildungsinstitutionen gleichsam vorgebaute Entree-Stationen mit Treffpunkt-Charakter, die den Bürgern beratend und kontaktvermittelnd zur Verfügung stehen:

"Lernservice-Studios könnte es in verschiedenen Größenordnungen und Aufbaustufen geben

- von einfachen Beratungsbüros bis zu ausgebauten Lernservice-Zentren mit

- Sprach- und Internetcafes,

- Lernateliers zur Präsentation anregender, auf verschiedene thematische Schwerpunkte bezogene Lernmöglichkeiten und Lernhilfen,

- Lernagenturen zur Experten- und Lernpartnervermittlung,

- Lernläden, in denen man neue Lerngeräte und Lernmaterialien kennenlernen, erproben, ausleihen, leasen oder kaufen kann usw."

Als ein Fazit darf festgehalten werden, dass Weiterbildungseinrichtungen auch zukünftig prägend für Bildungslandschaften sein werden. In lernenden Regionen und Städten werden Weiterbildungseinrichtungen jedoch einen stärkeren "Agenturcharakter" bekommen.

\section{LITERATUR}

CORCELIUS, F./DEDORATH, T./MÜLLER-SÄMANN, A.: Modularisierung der Bildungsarbeit. Wechselwirkungen zwischen Organisation und pädagogischem Personal. In: Klein, R./Reutter, G. (Hrsg.) 1998, S. 79-105.

DELPHI-BEFRAGUNG 1996/1998: Potenziale und Dimensionen der Wissensgesellschaft -Auswirkungen auf Bildungsprozesse und Bildungsstrukturen. Integrierter Abschlußbericht. München, Basel 1998.

DOHMEN, G. (Hrsg.): Selbstgesteuertes lebenslanges Lernen? Ergebnisse der Fachtagung des Bundesministeriums für Bildung, Wissenschaft, Forschung und Technologie vom 6.-7.12.1996 im Gustav-Stresemann-Institut. Bonn 1997.

DOHMEN, G.: Weiterbildungsinstitutionen, Medien, Lernumwelten, Rahmenbedingungen und Entwicklungshilfen für das selbstgesteuerte Lernen. Herausgegeben vom Bundesministerium für Bildung und Forschung. Bonn 1999.

DÖRING, N.: Lernen mit dem Internet. In: Issing, L./Klimsa, P. (Hrsg.) 1997, S. 305-335.

FRIEDRICH, H.F./MANDL, H.: Analyse und Förderung selbstgesteuerten Lernens. In: Weinert, F.E./Mandl, $\mathbf{H}$. 
(Hrsg.): Psychologie der Erwachsenenbildung. Enzyklopädie der Psychologie Bd. 4, Göttingen 1997, S. 237-293.

GABRIEL, N.: Kulturwissenschaften und Neue Medien. Darmstadt 1997

GÜNTHER, W./MANDL, H. (Hrsg.): Telelearning. Aufgabe und Chance für Bildung und Gesellschaft. Bonn: Telekom Multimedia Systemhaus 1997

HAACK, J.: Interaktivität als Kennzeichen von Multimedia und Hypermedia. In: Issing, L./Klimsa, P.(Hrsg.) 1997, S. 151-164.

HECKMAIR, B.: Vom Dozenten zum "Facilitator" - das Profil des Trainers im Umbruch. In: Grundlagen der Weiterbildung-Zeitschrift 1998, H. 4, S. 156-159.

HÜTHER, J.: Medienpädagogische Konzepte in der Bildungsarbeit mit Erwachsenen. Historische und aktuelle Aspekte der Kooperation von Medienpädagogik und Erwachsenenbildung. In: Hiegemann, S./Swoboda, W.H. (Hrsg.): Handbuch der Medienpädagogik, Opladen 1994, S. 289-302.

ISSING, L./KLIMSA, P. (Hrsg.): Information und Lernen mit Multimedia. Weinheim 1997.

ISSING, L./KLIMSA, P. Multimedia - Eine Chance für Information und Lernen. In: Issing/Klimsa (Hrsg.) 1997 S. 1-3.

KADE, J.: Entgrenzung und Entstrukturierung. Zum Wandel der Erwachsenenbildung in der Moderne. In: Derichs-Kunstmann, K./Faulstich, P./Tippelt, R. (Hrsg.): Entraditionalisierung der Erwachsenenbildung. Dokumentation der Jahrestagung 1996 der Kommission Erwachsenenbildung der Deutschen Gesellschaft für Erziehungswissenschaft, Frankfurt a. M. 1997, S. 13-31.

KLEIN, R./REUTTER, G. (Hrsg.): Lehren ohne Zukunft? Wandel der Anforderungen an das pädagogische Personal in der Erwachsenenbildung. Baltmannsweiler 1998.

KRUG, P.: Selbstgesteuertes Lernen im Prozeß des lebenslangen Lernens. In: Dohmen, G. (Hrsg.) 1997, S. 84-89.
NIEGEMANN, H.: Multimedia in der Weiterbildung. Design- und Organisationsfragen. In: Vogel, N. (Hrsg.): Organisation und Entwicklung in der Weiterbildung. Bad Heilbrunn 1998, S. 162-185.

NUISSL VON REIN, E.: Institutionen im lebenslangen Lernen. In: FaulstichWieland, H./Nuissl von Rein, E./Siebert, H./Weinberg, J. (Hrsg.): Lebenslanges Lernen - Selbstorganisiert? Literatur- und Forschungsreport Weiterbildung Nr. 39. Frankfurt a. $\mathbf{M}$. 1997, S. 41-49.

VON REIN, ANTJE (Hrsg.): Medienkompetenz als Schlüsselbegriff. Bad Heilbrunn 1996.

SCHÖLL, I./PASSENS, B.: Selbstgesteuertes Lernen in der Diskussion. In: Klein, R./Reutter, G. (Hrsg.) 1998, S. 153-173.

SIEBERT, H.: Pädagogischer Konstruktivismus. Neuwied 1999.

STADELHOFER, C.: Selbstgesteuertes Lermen und Neue Kommunikationstechnologien. In: Dohmen, G (Hrsg.) 1999 , S. 147-208.

STANG, R./HAGEDORN, F./NISPEL, A.: Fortbildungsmodule "Multimedia in der Erwachsenenbildung". Bausteine für Fortbildungsangebote. In: Nispel, A./Stang, R. /Hagedorn, F. (Hrsg.): Pädagogische Innovation mit Multimedia 2. Organisation und Fortbildung. Frankfurt 1998, S. 121-135.

TIETGENS, H.: Aspekte der Professionalität der ErwachsenenbildnerInnen. In: Klein, R./Reutter, G. (Hrsg.) 1998, S. $39-45$.

WEBER, K.: Selbstgesteuertes Lernen. Ein Konzept macht Karriere. In: Grundlagen der WeiterbildungZeitschrift 1996, H. 4, S. 178-182.

WEIDENMANN, B.: Multicodierung und Multimodalität im Lernprozeß. In: Issing/Klimsa. (Hrsg.) 1997, S. 65-81.

WEINBERG, J.: Vorwort. Anforderungen an das erwachsenenpädagogische Aufgaben- und Selbstverständnis. In: Klein, R./Reutter, G. (Hrsg.) 1998, S. 1-12. 


\section{RESUMEN}

Aun cuando se ve la "promesa" de los nuevos medios con escepticismo, probablemente tendrán grandes consecuencias en las áreas de aprendizaje. Los nuevos medios serán dirigidos hacia un amplio perfil de exigencias de los protagonistas centrales en el aprendizaje, los estudiantes, los profesores y las instituciones. Esto exige de la educación de adultos una aclaración de naturaleza propia.

Los escenarios de "la sociedad de conocimientos y saber" serán sacados a partir de un aprendizaje independiente, el cual, es en alto grado una adquisición de conocimientos activos, los cuales si bien encuentran preguntas relevantes también encuentra una dirección de respuestas propias. ¿Qué competencias de autoaprendizaje necesita el estudiante y cómo las consigue? También se cambia la forma en que se entiende la profesión del profesor. En lugar de estar el docente y los entrenadores como suministradores de conocimientos, es un aprendizaje compartido, el proceso de aprendizaje incita y aconseja al estudiante a ser un explorador. Aunque el aprendizaje autodirigido a primera vista implica una fuerte autoresponsabilidad del estudiante, no significa que las instituciones llegarán a sobrar, pero si deben desarrollar un cambio y un complemento en las relaciones para las formas de aprendizaje informales.

Este aporte se dirige especialmente sobre los "Aspectos de Relación" de los nuevos medios. ¿Qué consecuencias tienen entonces para larelación jerárquica entre estudiantes y profesores? ¿Cómo se transformaran los medios el rol/papel entre los estudiantes y los profesores?

En conclusión se hace referencia a las debilidades de la filosofía del aprendizaje tradicional y también al potencial de los nuevos medios para una nueva cultura del estudiante.

\section{ABSTRACT}

Auch wenn man den Verheißungen neuer Medien mit Skepsis begegnet, so werden sie vermutlich weitreichende Konsequenzen für den Bereich des Lernens und Lehrens haben. Die neuen Medien werden zu einem erweiterten Anforderungsprofil der zentralen Akteuren im Lerngeschehen, den Lermenden, den Lehrenden und den Institutionen, führen. Dies verlangt von der Erwachsenenbildung eine Klärung des eigenen Selbstverständnisses.

In den Szenarien der Wissensgesellschaft wird von einem selbständigen Lernenden ausgegangen, der hochgradig aktiv bei der Aneignung von Wissen ist; der sowohl die relevanten Fragen selbst stellt als auch in eigener Regie Antworten 
darauf findet. Welche Selbstlemkompetenzen benötigen die Lemenden und wie erlangen sie diese? Aber auch das Professionsverständnis der Lehrenden ändert sich. Anstelle des Dozenten und Trainers als Sender von Wissen tritt der Lernbegleiter, der Lemprozesse anregt und beratend dem Lernenden als Entdeckungseisenden zur Seite steht. Auch wenn das selbstgesteuerte Lernen auf den ersten Blick eine stärkere Eigenverantwortlichkeit der Lernenden impliziert, bedeutet dies nicht, dass Bildungsinstitutionen überflüssig werden, aber sie müssen komplementäre Wechsel- und Ergänzungsbeziehungen zu informelle Lernformen entwickeln.

In diesem Beitrag wird insbesondere auf den "Beziehungsaspekt" der neuen Medien gezielt. Wenn sich Aus- und Weiterbildner zunehmend weniger als Wissensvermittler sehen, dann ist dies auch für hierarchische Verhältnis zwischen Lehrenden und Lernenden nicht folgenlos Wie wird sich das pädagogische Rollenmuster zwischen Lehrenden und Lernenden wandeln?

Abschließend wird auf die Schwächen der traditionellen Lernphilosophie und auf das Potential der neuen Medien für eine neue Kultur des Lernens hingewiesen. 